\title{
Aquaporin 1-expressing MCF-7 mammary carcinoma cells show enhanced migration in vitro
}

\author{
Yong Jiang ${ }^{1,2}$, Zhi-Bin Jiang ${ }^{3}$ \\ ${ }^{1}$ Key Laboratory of Industrial Microbiology, Ministry of Education, Tianjin University of Science and Technology, Tianjin, China; \\ ${ }^{2}$ College of Biotechnology, Tianjin University of Science and Technology, Tianjin, China; \\ ${ }_{3}^{3}$ Jilin Combined Traditional Chinese and Western Medicine Hospital in Jilin Province, Jilin, China. \\ Email:jiangyong@tust.edu.cn
}

Received 6 September 2009; revised 19 October 2009; accepted 20 October 2009.

\begin{abstract}
Recent studies have demonstrated that aquaporin (AQP) expression facilitates cell migration and promotes angiogenesis and neutrophil motility. Migration of tumor cells is a crucial step in tumor invasion and metastasis. Here we investigated the expression of AQP in MCF-7 human mammary carcinoma cells and characterized its function in cell migration. Reverse Transcription-Polymerase Chain Reaction, Immunoblot and Immunofluorescence analysis demonstrated two populations of MCF-7 cell clones with low (AQP1L) and high (AQP1H) AQP1 expression and the AQP1 protein expression patterns in the plasma membrane of MCF-7 cells. MCF-7 cell clones (AQP1L and AQP1H) with low and about two-fold higher osmotic water permeability were identified by functional assays with corresponding low and high AQP1 expression. Cell migration rate was remarkably higher in AQP1H cells as compared to AQP1L cells, assessed by wound healing and transwell migration assays. Adenoviral-mediated mRNA and protein expression of AQP1 in AQP1L cells increased their water permeability and migration rate to the level similar to AQP1H cells. The results provided direct evidence that aquaporin-mediated plasma membrane water permeability played an important role in mammary carcinoma cell migration and may be associated with mammary carcinoma invasion and metastasis.
\end{abstract}

Keywords: Aquaporin; Water Permeability; Cell Migration

\section{INTRODUCTION}

Since Agre laboratory discovered the first aquaporin [1] from red blood cells, thirteen homologous members (AQP0-AQP12) in mammals and hundreds of AQPs in other species have been identified molecularly $[2,3]$. Aquaporins (AQPs) are membrane water channels that play pivotal roles in physiological and pathophysiological processes of diverse mammalian organs $[4,5,6]$. Recent studies indicated a novel role of AQPs in cell migration. Mice lacking AQP1, the endothelial water channel, were found to have impaired endothelial cell migration and tumor angiogenesis [7]. In the same study, over-expression of AQP1 or AQP4 (a structurally related water channel) was also found to promote migration of non-endothelial cells. Subsequent studies demonstrated that AQP1 expression promoted the migration of epithelial cells of kidney proximal tubules and facilitated wound healing of injured proximal tubules [8]. In the brain, AQP4 deletion in astroglial cells was also found to result in impaired migration and remarkably reduced glial scar formation after stab injury [9]. In addition, some other studies provided evidence that aquaglyceroporin AQP9 may be involved in neutrophil cell motility $[10,11]$. These studies suggested that aquaporin-Mediated plasma membrane water permeability may be a fundamental determinant of cell migration and associated physiological and pathological processes. Migration of tumor cells is a crucial step in tumor invasion and metastasis $[12,13]$. Previous studies demonstrated the expression of AQPs in some types of tumor cells $[14,15,16]$.

We hypothesized that AQPs also play a critical role in mammary carcinoma cell migration. In the present study, we provided the first evidence for the involvement of AQP1 in mammary carcinoma cell migration.

\section{MATERIALS AND METHODS}

\subsection{Cell Culture}

MCF-7 human mammary carcinoma cells (from Cell Bank of Shanghai Institute of Biochemistry and Cell Biology) were maintained in DMEM supplemented with $10 \% \mathrm{FBS}, 100 \mu \mathrm{g} / \mathrm{mL}$ penicillin and $100 \mu \mathrm{g} / \mathrm{mL}$ streptomycin. The cells were plated in 6-well plates 
and maintained at $37^{\circ} \mathrm{C}$ and $5 \% \mathrm{CO}_{2}$. The monoclonal MCF-7 mammary carcinoma cell line was isolated and expanded by limited dilution to investigate the expression and function of AQP1.

\subsection{Reverse Transcription-Polymerase Chain Reaction}

Total RNA was extracted from the cultured MCF-7 human mammary carcinoma cells with RNeasy micro kit (QIAGEN) and cDNA was reversely transcribed (RT) from the total RNA using a superscript firststrand synthesis system (invitrogen). The cDNA was used as a template for polymerase chain reaction (PCR) amplification $\left(94^{\circ} \mathrm{C}, 5 \mathrm{~min}\right.$ and then 30 cycles of $94^{\circ} \mathrm{C}$ for $30 \mathrm{~s} ; 60^{\circ} \mathrm{C}$ for $30 \mathrm{~s} ; 72^{\circ} \mathrm{C}$ for $60 \mathrm{~s}$ ) using primers flanking a $252 \mathrm{bp}$ fragment of AQP1 (sense, 5'-CTCTCTgTAgCCCTTggACACCTC-3'; antisense, 5'-ggCATCCAggTCATACTCCTCCAC-3') and flanking a $213 \mathrm{bp}$ fragment of GAPDH (sense, 5'-ATTCAACGGCACAGTCAAGG-3'; antisense, 5'- GCAGAAGGGGCGGAGATGA-3'). The PCR products were analyzed by agarose gel electrophoresis.

\subsection{Immunoblotting}

MCF-7 cells were washed with phosphate-buffered saline (PBS), incubated with $2 \mathrm{~mL} 50 \mathrm{mM} \mathrm{Na} \mathrm{N}_{2}$-EDTA solution for 5 to $10 \mathrm{~min}$ at room temperature and solubilized at $60^{\circ} \mathrm{C}$ for $15 \mathrm{~min}$ in Laemmli sample buffer. Equal amounts of protein $(10 \mu \mathrm{g})$ were resolved by SDS-PAGE on $12 \%$ polyacrylamide gel, blotted to polyvinylidene difluoride (PVDF) membranes, blocked with $5 \%(\mathrm{w} / \mathrm{v})$ nonfat milk and washed in Tris-buffered saline (TBS)-Tween solution. Primary antibodies (anti-AQP1, anti-Tubulin) (Chemicon, $1: 1000$ ) and secondary antibody conjugated to horseradish peroxidase (Sigma, 1:3000) were applied successively and detected by enhanced chemoluminescence (Amersham).

\subsection{Immunofluorescence}

MCF-7 cells were seeded on coverglasses $24 \mathrm{~h}$ before fixation in PBS supplemented with 4\% paraformaldehyde (PFA) for $15 \mathrm{~min}$. Cells were washed with PBS, blocked and permeabilized by incubation in PBS containing $2 \%(\mathrm{w} / \mathrm{v})$ bovine serum albumin (Sigma), and $0.1 \%(\mathrm{v} / \mathrm{v})$ Triton $\mathrm{X}-100$ for 30 mins. Cells were then exposed to a polyclonal rabbit anti-rat AQP1 antibody (Chemicon, 1:1000) overnight at $4^{\circ} \mathrm{C}$. After washing, a Cy-3-conjugated anti-rabbit $\mathrm{IgG}$ secondary antibody (Sigma) was applied at 1:500 for $1 \mathrm{~h}$ at room temperature. Cells were washed with PBS and visualized by fluorescence microscopy (Olympus).

\subsection{Adenovirus-Mediated AQP1 Expression}

The recombinant adenovirus expressing human AQP1 (Ad-AQP1) and control $\beta$-galactosidase adenovirus (Ad-lacZ) were generated using the ViraPower adenoviral expression system (Invitrogen) according to the manufacturer's instruction and purified/ concentrated with an Adeno-X virus purification kit (BD Biosciences-Clontech). The MCF-7l cells were infected with Ad-AQP1 or Ad-lacZ (control adenovirus) at a concentration of $150 \mathrm{PFU} /$ cell respectively. AQP1 expression and function were analyzed at $48 \mathrm{~h}$ after infection.

\subsection{Water Permeability Measurements}

The osmotic water permeability of MCF-7 cell plasma membrane was measured by a calcein fluorescence quenching method described previously [17], with modifications. Briefly, MCF-7 cells were grown on round glass coverslips pre-coated with polylysine for $24 \mathrm{~h}$. The cells were incubated with $10 \mu \mathrm{mol} / \mathrm{L}$ calcein-AM (Molecular probes) for $10 \mathrm{~min}$ and then mounted in a perfusion chamber designed for rapid solution exchange. The time-course of cytoplasmic calcein fluorescence in response to an osmotic gradient was monitored by exchanging perfusate osmolality between $300 \mathrm{mOsmol}$ (PBS) and $150 \mathrm{mOsmol}$ (diluted with distilled water). The rate of change of cell volume was presented as reciprocal exponential time constant $(1 / \tau)$, which is proportional to osmotic water permeability, where $\tau$ is the time needed from the beginning of osmotic switch to the point when the cytoplasmic calcein fluorescence reaches its maximum.

\subsection{In Vitro Wound Healing Assay}

Assays were performed as described previously with modification [7]. Briefly, MCF-7 cells were cultured as confluence monolayers on 6-well plates and synchronized in $1 \%$ fetal bovine serum for $24 \mathrm{~h}$. The monolayers were wounded by removing a $300-500$ $\mu \mathrm{m}$ strip of cells across the well with a $200 \mu \mathrm{L}$ pipette tip and then washed twice to remove non-adherent cells. Wound healing was quantified as the average linear speed of the wound edges over $24 \mathrm{~h}$.

\subsection{Transwell Migration Assays}

Invasive cell migration was measured using a modified Boyden chamber chemotaxis assay. MCF-7 cells were seeded on top of a culture plate insert (Corning Costar) containing a polycarbonate filter $(6.5 \mathrm{~mm}$ diameter, $8 \mu \mathrm{m}$ pores) pre-coated with fibronectin $(0.5 \mathrm{mg} / \mathrm{mL})$. The upper chamber contained cells in 


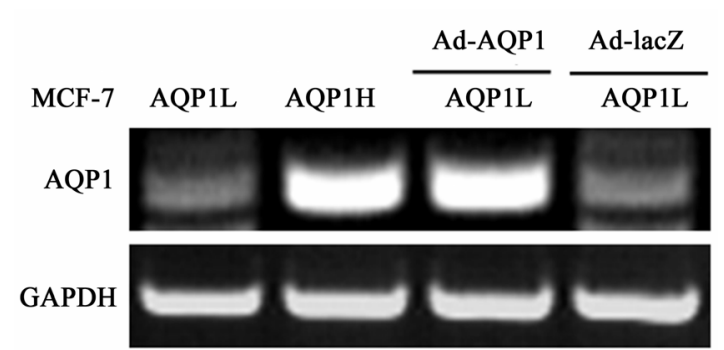

(a)

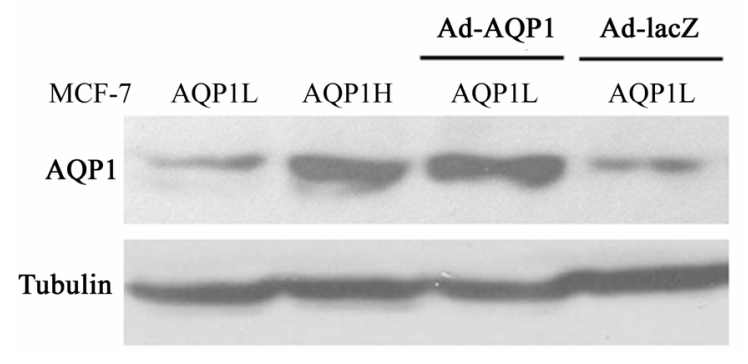

(b)

Figure 1. The expression of AQP1 in MCF-7 mammary carcinoma cells. (a) Adenovirus-mediated increased mRNA expression of AQP1 in MCF-7 cells. RT-PCR analysis of AQP1 in clones (AQP1L, AQP1H, AQP1L-Ad-AQP1 and AQP1L-Ad-lacZ). (b) Adenovirus-mediated increased protein expression of AQP1 in MCF-7 cells. Immunoblot analysis showing AQP1 protein expression in clones (AQP1L, AQP1H, AQP1L-Ad-AQP1 and AQP1L-Ad-lacZ).

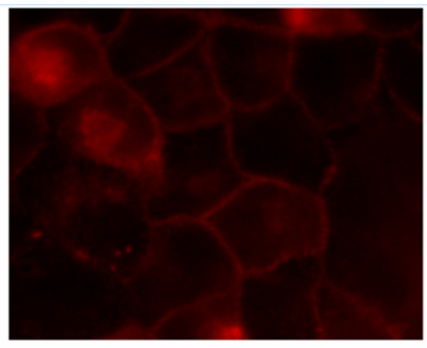

AQP1L

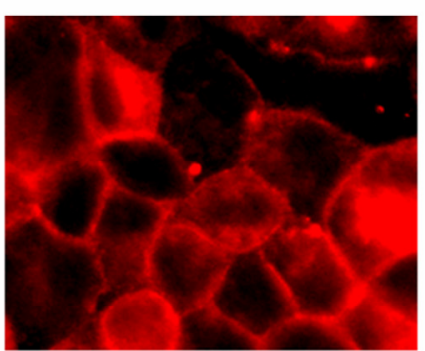

$\frac{\text { Ad-AQP1 }}{\text { AQP1L }}$

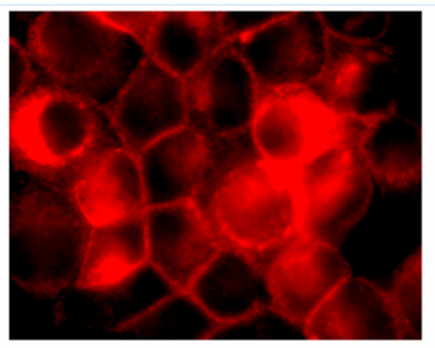

AQP1H

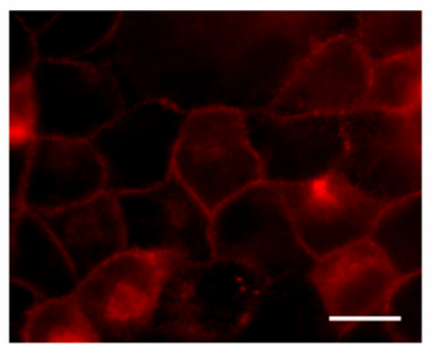

Ad-lacZ

AQP1L

Figure 2. Immunofluorescence showing AQP1 expression in plasma membrane of AQP1L, AQP1H, AQP1L-Ad-AQP1 and AQP1L-Ad-lacZ MCF-7 cells. Scale bar $=10 \mu \mathrm{m}$.

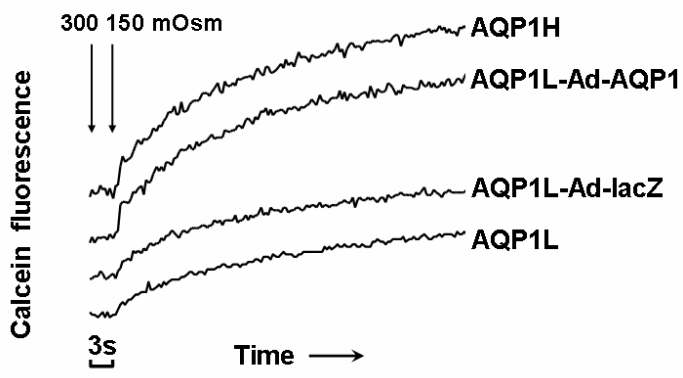

(a)

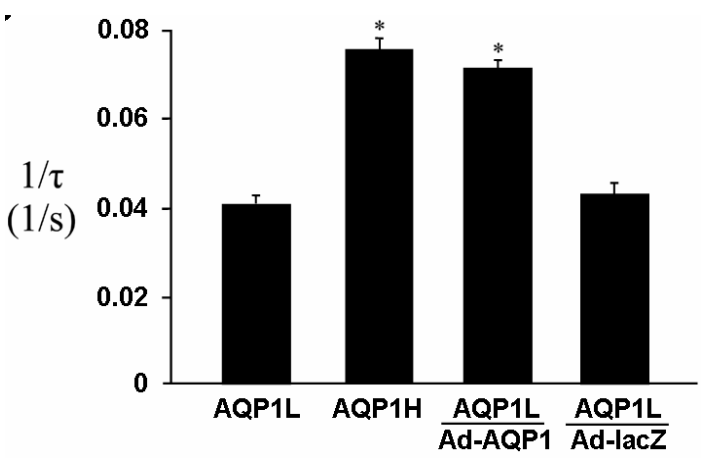

(b)

Figure 3. MCF-7 cell water permeability analysis. A, Representative curves showing time-course of calcein fluorescence in response to changes in perfusate osmolality from 300 to $150 \mathrm{mOsmol}$. B, Cell swelling rates presented by reciprocal exponential time constants $(1 / \tau)$ in six sets of experiments, $(\mathrm{n}=6$, mean $\pm \mathrm{SE}$, Asterisks denote $P<0.01$ versus AQP1L cells). 

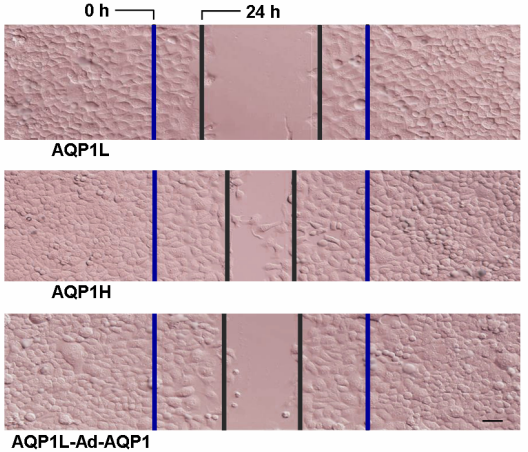

(a)

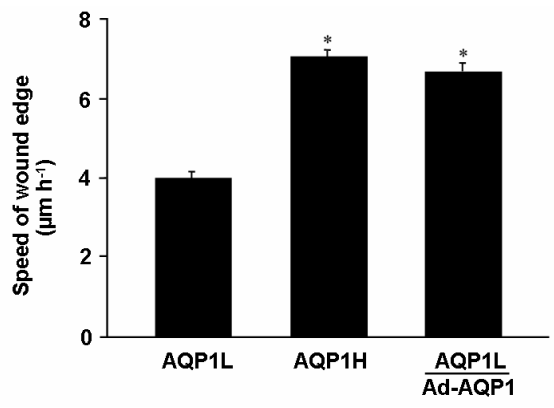

(b)

Figure 4. AQP1 facilitated wound healing of MCF-7 cell monolayers. A, Representative images of wound closure showing initial wound edge and wound edge after $24 \mathrm{~h}$. Scale bar $=20 \mu \mathrm{m}$. B, The quantified wound edge speed ( $\mathrm{n}=6$, mean $\pm \mathrm{SE}$, Asterisks denote $P<0.01$ versus AQP1L cells).
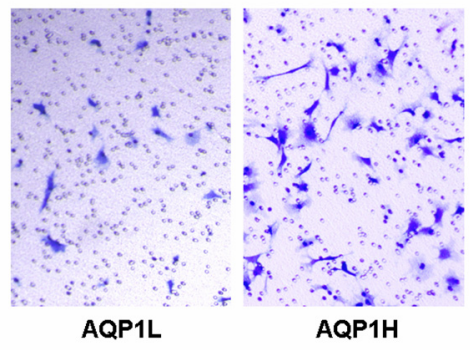

(a)

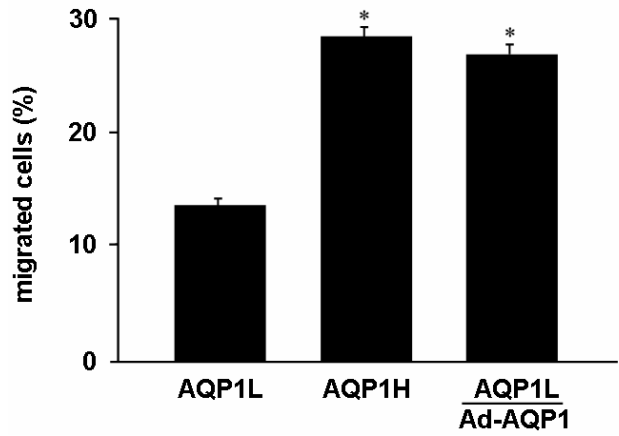

(b)

Figure 5. AQP1 promoted invasive migration of MCF-7 cells. A, AQP1L and AQP1H cells migrated through the porous transwell filter. Infection of Ad-AQP1 in AQP1L cells enhanced the transwell migration rate of cells to the level similar to AQP1H cells. Cells were stained with Coomassie blue. Scale bar $=10 \mu \mathrm{m}$. B, Percentage of migrated cells at $12 \mathrm{~h}$ after plating ( $\mathrm{n}=6$, mean $\pm \mathrm{SE}$, Asterisks denote $P<0.01$ versus AQP1L cells).

DMEM plus $1 \%$ fetal bovine serum, and the lower chamber contained DMEM plus $10 \%$ (chemoattractant). Cells were incubated for $12 \mathrm{~h}$ at $37^{\circ} \mathrm{C}$ and $5 \%$ $\mathrm{CO}_{2}$. Cells that did not migrate were wiped away from the top of transwell filter and migrating cells on the bottom surface were counted after staining with Coomassie blue.

\subsection{Statistical Analysis}

Data were expressed as mean $\pm \mathrm{SE}$ and accompanied by the number of experiments performed independently, and analyzed by t-test. Differences at $\mathrm{P}<0.05$ were considered statistically significant.

\section{RESULTS}

\subsection{Expression of AQP1 in Two Populations of MCF-7 Mammary Carcinoma Cells}

We generated monoclonal MCF-7 mammary carcinoma cell line by limited dilution. Selective expression of AQP1 was identified by RT-PCR analysis using primers specific amplifying fragments of human AQP1. Interestingly, two populations of MCF-7 cell clones with low (AQP1L) and high (AQP1H) AQP1 expression were identified by RT-PCR analysis. The recombinant adenovirus expressing human AQP1 (Ad-AQP1) increased expression of AQP1 in AQP1L cells versus control adenovirus (Ad-lacZ) infected cells (Figure 1(a)). Immunoblot and immunofluorescence using a AQP1 polyclonal antibody confirmed the same AQP1 expression patterns as shown in Figure $1 \mathrm{~B}$ and Figure 2. Immunofluorescence indicated expression of AQP1 protein in the plasma membrane of MCF-7 cells (Figure 2). The AQP1L and AQP1H cells possessed identical protein pattern on SDSPAGE (data not shown). The identical protein pattern of the two cell populations indicated that they were from the same parental cell type except that the AQP1 expression was different.

\subsection{Expression of AQP1 Confered High Water Permeability of MCF-7 Cells}

Osmotic water permeability of MCF-7 cell plasma membrane was measured by a calcein fluorescence quenching method. Figure 3 showed the results of 
water permeability measurements of MCF-7 cell lines. The two populations of MCF-7 cell clones (AQP1L and $\mathrm{AQP} 1 \mathrm{H}$ ) with low and about two-fold higher osmotic water permeability with corresponding low and high AQP1 expression were identified by functional assay (Figure 3(a), 3(b)). To determine whether AQP1 expression accounted for the increased water permeability of AQP1L cells, we overexpressed AQP1 in AQP1L cells. Accordingly, osmotic water permeability was increased by about 1.7 fold in AQP1L cells infected with Ad-AQP1. In the same experimental conditions, infection with Ad-lacZ did not increase plasma membrane water permeability of AQP1L cells.

\subsection{Expression of AQP1 Facilitated Wound Healing of MCF-7 Cell Monolayers}

Wound closure experiments were performed to confirm the role of AQP1 in MCF-7 mammary carcinoma cell migration. Figure 4 showed significantly accelerated wound closure in AQP1H cells and AQP1L cells infected with Ad-AQP1 at concentration of 150 $\mathrm{PFU} /$ cell as compared to AQP1L cells.

\subsection{Expression of AQP1 Promoted Invasive Mi- gration of MCF-7 Cells}

Transwell migration assay using a modified Boyden chamber was performed as an in vitro model of invasive migration of $\mathrm{AQP} 1 \mathrm{~L}$ and $\mathrm{AQP} 1 \mathrm{H}$ cells, and AQP1L cells infected with Ad-AQP1. As shown in Figure 5, migration rate of $\mathrm{AQP} 1 \mathrm{H}$ cells towards $10 \%$ FBS through the $8 \mu \mathrm{m}$ pores in the transwell filter was significantly higher $(\sim 2.1$ fold $)$ than that of AQP1L cells. Infection of Ad-AQP1 at concentration of 150 $\mathrm{PFU} /$ cell enhanced the transwell migration rate of AQP1L cells to the level similar to AQP1H cells, suggesting an important role of AQP1 in facilitating cell migration.

\section{DISCUSSION}

Migration of tumor cells through narrow extracellular spaces requires cell volume regulation and shape change. Previous studies revealed that ion channels and transporters were essential for cell volume regulation and invasive migration of some human tumors $[18,19]$. Our present study demonstrated for the first time that aquaporin-mediated transmembrane water flux was an important determinant of MCF-7 mammary carcinoma cell migration and may be associated with mammary carcinoma invasion and metastasis. We proposed that transmembrane water efflux through aquaporin water channels facilitated cell volume change mediated by net salt efflux through ion channels and transporters in the mi- grating mammary carcinoma cells, leading to enhanced motility and invasion. There were evidence suggesting a role of AQPs in tumor cell migration and metastasis. Expression of several AQPs was found in a wide range of tumor cells in addition to the ubiquitous expression of AQP1 in the microvessels of solid tumors $[14,15,16]$. Warth et al. [16] found that AQP4 expression at the endfeet membranes was redistributed in high-grade astrocytomas.

Our experiments provided direct evidence that aquaporin-mediated high plasma membrane water permeability contributed to cell shape-volume change required for mammary carcinoma cell migration. Cell migration rate was remarkably higher in $\mathrm{AQP} 1 \mathrm{H}$ cells as compared to AQP1L cells in both wound healing and invasive transwell migration assays. Adenoviralmediated AQP1 expression in AQP1L cells increased their plasma membrane water permeability and migration rate, further supporting the role of AQP1 in facilitating migration of the mammary carcinoma cells. Future studies are needed to uncover the role of aquaporin water channels in vivo tumor cell migration and metastasis.

\section{ACKNOWLEDGEMENT}

This work was supported by the National Natural Science Foundation of China (Grant No.30800561), Tianjin Natural Science Foundation (GrantNo.07JCYBJC16400), and Scientific Research Foundation of Tianjin University of Science and Technology (GrantNo.20090402).

\section{REFERENCES}

[1] Preston, G.M., Carroll, T.P., Guggino, W.B. and Agre P. (1992) Appearance of water channels in Xenopus oocytes expressing red cell CHIP 28 protein. Science, 256, 385-387.

[2] Agre P. (2006) The aquaporin water channels. Proc Am Thorac Soc, 3, 5-13.

[3] Takata, K., Matsuzaki, T. and Tajika, Y. (2004) Aquaporins: water channel proteins of the cell membrane. Prog Histochem Cytochem, 39, 1-83.

[4] Agre, P., King, L.S., Yasui, M., Guggino, W.B. and Ottersen, O.P. (2002) Aquaporin water channels-from atomic structure to clinical medicine. J Physiol, 542, 3-16.

[5] Verkman, A.S. (2002) Physiological importance of aquaporin water channels. Ann Med, 34, 192-200.

[6] Verkman, A.S. (2005) More than just water channels: unexpected cellular roles of aquaporins. J Cell Sci, 118, 3225-3232.

[7] Saadoun, S., Papadopoulos, M.C., Chikuma, M.H. and Verkman, A.S. (2005) Impairment of angiogenesis and cell migration by targeted aquaporin-1 gene disruption Nature, 434, 786-792.

[8] Chikuma, M.H. and Verkman, A.S. (2006) Aquaporin-1 facilitates epithelial cell migration in kidney proximal tubule. J Am Soc Nephrol, 17, 39-45.

[9] Saadoun, S., Papadopoulos, M.C., Watanabe ,H., Yan, D. and Manley G.T. (2005) Involvement of aquaporin-4 in 
astroglial cell migration and glial scar formation. $J$ Cell Sci, 118, 5691-5698.

[10] Loitto, V.M., Forslund, T., Sundqvist, T., Magnusson, K. E. and Gustafsson, M. (2002) Neutrophil leukocyte motility requires directed water influx, $J$ Leukoc Biol, 71, 212-222.

[11] Loitto, V.M. and Magnusson, K.E. (2004) $\mathrm{Hg}^{2+}$ and small-sized polyethylene glycols have inverse effects on membrane permeability, while both impair neutrophil cell motility. Biochem Biophys Res Commun, 316, 370378.

[12] Bogenrieder, T. and Herlyn, M. (2003) Axis of evil: molecular mechanisms of cancer metastasis. Oncogene, 22, 6524-6536.

[13] Harlozinska, A. (2005) Progress in molecular mechanisms of tumor metastasis and angiogenesis. Anticancer Res, 25, 3327-3333.

[14] Yang, J.H., Shi, Y.F., Cheng, Q. and Deng, L. (2006) Expression and localization of aquaporin-5 in the epithelial ovarian tumors. Gynecol Oncol, 100, 294-299.
[15] Mazal, P.R., Susani, M., Wrba, F. and Haitel, A. (2005) Diagnostic significance of aquaporin-1 in liver tumors. Hum Pathol, 36, 1226-1231.

[16] Warth, A., Mittelbronn, M. and Wolburg, H. (2005) Redistribution of the water channel protein aquaporin-4 and the $\mathrm{K}^{+}$channel protein Kir4.1 differs in low-and high-grade human brain tumors. Acta Neuropathol, 109, 418-426.

[17] Solenov, E., Watanabe, H., Manley, G.T. and Verkman, A. S. (2004) Sevenfold-reduced osmotic water permeability in primary astrocyte cultures from AQP-4-deficient mice, measured by a fluorescence quenching method. Am $J$ Physiol Cell Physiol, 286, 426-432.

[18] Ransom, C.B., O'Neal, J.T. and Sontheimer, H. (2001) Volume-activated chloride currents contribute to the resting conductance and invasive migration of human glioma cells. J Neurosci, 21, 7674-7683.

[19] Soroceanu, L., Manning, T.J. and Sontheimer, H. (1999) Modulation of glioma cell migration and invasion using $\mathrm{Cl}^{-}$ and $\mathrm{K}^{+}$ion channel blockers. $J$ Neurosci, 19, 5942-5954. 